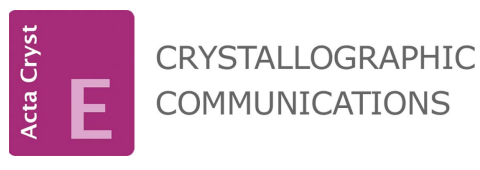

ISSN 2056-9890

Received 22 March 2017

Accepted 24 April 2017

Edited by A. J. Lough, University of Toronto, Canada

Keywords: crystal structure; dibromomethoxyseseline (DBMS); seseline: bromination; bromo product; $\pi-\pi$ stacking; $\mathrm{C}-\mathrm{H}$. . O interactions.

CCDC reference: 1545541

Supporting information: this article has supporting information at journals.iucr.org/e

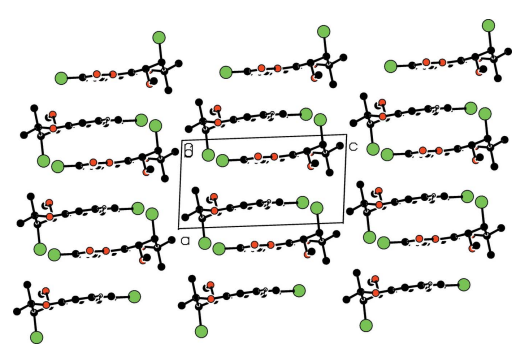

OPEN $\odot$ ACCESS

\section{Crystal structure of dibromomethoxyseselin (DBMS), a photobiologically active pyran- ocoumarin}

\author{
A. K. Bauri, ${ }^{\text {a }}$ Sabine Foro ${ }^{\mathrm{b}}$ and A. F. M. M. Rahman ${ }^{\mathrm{c} *}$
}

a Bio-Organic Division, Bhabha Atomic Research Centre, Trombay, Mumbai 400 085, India, ${ }^{\mathbf{b}}$ Institute of Materials Science, Darmstadt University of Technology, Alarich-Weiss-Strasse 2, D-64287 Darmstadt, Germany, and ${ }^{\mathrm{c}}$ Department of Applied Chemistry \& Chemical Engineering, University of Dhaka, Dhaka-1000, Bangladesh. *Correspondence e-mail: mustafizacce@du.ac.bd

The title compound, $\mathrm{C}_{15} \mathrm{H}_{14} \mathrm{Br}_{2} \mathrm{O}_{4}$ [systematic name: rac-(9S,10R)-3,9-dibromo10-methoxy-8,8-dimethyl-9,10-dihydropyrano[2,3- $h]$ chromen-2( $8 H)$-one], is a pyranocoumarin derivative formed by the bromination of seselin, which is a naturally occurring angular pyranocoumarin isolated from the Indian herb Trachyspermum stictocarpum. In the molecule, the benzopyran ring system is essentially planar, with a maximum deviation of 0.044 (2) $\AA$ for the O atom. The dihydropyran ring is in a half-chair conformation and the four essentially planar atoms of this ring form a dihedral angle of $4.6(2)^{\circ}$ with the benzopyran ring system. In the crystal, molecules are linked by weak $\mathrm{C}-\mathrm{H} \cdots \mathrm{O}$ hydrogen bonds, forming chains propagating along [010]. In addition, $\pi-\pi$ stacking interactions, with centroid-centroid distances of 3.902 (2) and 3.908 (2) $\AA$, link the hydrogenbonded chains into layers parallel to (001).

\section{Chemical context}

The title compound is a substituted product of seselin containing two bromine atoms and a methoxy group. This class of pyranocoumarins have an absorption band in the near-UV region due to the presence of extended conjugated double bonds and exhibit photomutagenic (Appendino et al., 2004) and photocarcinogenic properties to bind with the purin base of DNA in a living cell to yield photoadducts (Conforti et al., 2009). Based on the properties of these molecules, they are employed for the treatment of numerous inflammatory skin diseases such as atopic dermatitis and the pigment disorders vitiligo and psoriasis on exposure to ultra violet (UV) radiation in photodynamic therapy (PDT). It has also been found that as a result of their strong ability for absorption of UV radiation, they are utilized as photoprotective agents to prevent the absorption of harmful UV radiation by the skin in the form of a variety of sun-screening lotions widely used in dermatological applications in the cosmetic and pharmaceutical industries (Chen et al., 2007, 2009). In addition to these activities, antiproliferative activity and photo-toxicity of related coumarin molecules has been reported against numerous cancer cell lines such as HL60, A431 (Conconi et al., 1998). Inhibited proliferation in the human hepatocellular carcinoma cell line has also been reported (March et al., 1993). Recently, this type of molecule has been connected as a spacer with porphyrin moieties to obtain a scaffold-type macromolecule (molecular nanotweezers) and has been employed to study the interaction (host-guest interaction) with fullerenes 
such as $\mathrm{C}_{60}$ and $\mathrm{C}_{70}$ (Banerjee et al., 2014; Ghosh et al., 2014) in supramolecular chemistry and material science. Molecular tweezers containing a coumarin moiety showed better quantum yield and fluorescence absorption as a result of the presence of the extended conjugated enone of pyranocoumarin. As part of our ongoing studies in this area, we herein describe the synthesis and structure of the title molecule.

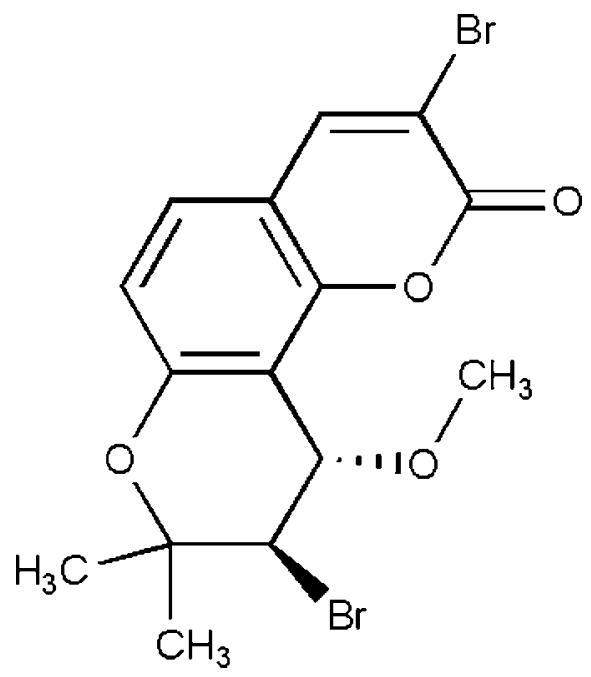

\section{Structural commentary}

The title molecule (Fig. 1) is composed of three different types of rings viz. benzene, pyran and dihydropyran. The benzopyran ring system $\mathrm{C} 1 / \mathrm{C} 5-\mathrm{C} 12 / \mathrm{O} 2$ is essentially planar with a maximum deviation of 0.044 (2) $\AA$ for atom O2. The dihydropyran ring $\mathrm{C} 1-\mathrm{C} 5 / \mathrm{O} 1$ is in a half-chair conformation and atoms C2 and C3 deviate by -0.385 (4) and 0.280 (4) $\AA$ from the plane through the other four essentially planar atoms (mean deviation $0.003 \AA$ ), which makes a dihedral angle of $4.6(2)^{\circ}$ with the benzopyran ring system. The relative stereochemistry at atoms $\mathrm{C} 3$ and $\mathrm{C} 4$ is $R / S$ and $S / R$.

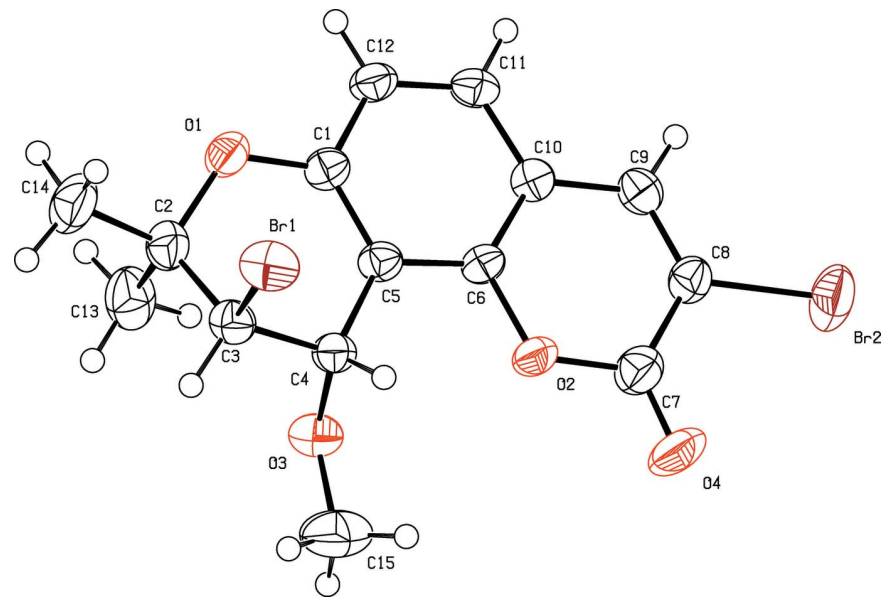

Figure 1

The molecular structure of the title compound, showing the atom labelling and displacement ellipsoids drawn at the $50 \%$ probability level
Table 1

Hydrogen-bond geometry $\left(\AA{ }^{\circ}\right)$.

\begin{tabular}{lllll}
\hline$D-\mathrm{H} \cdots A$ & $D-\mathrm{H}$ & $\mathrm{H} \cdots A$ & $D \cdots A$ & $D-\mathrm{H} \cdots A$ \\
\hline $\mathrm{C} 11-\mathrm{H} 11 \cdots \mathrm{O} 4^{\mathrm{i}}$ & 0.93 & 2.57 & $3.188(6)$ & 124 \\
\hline
\end{tabular}

Symmetry code: (i) $x, y-1, z$.

\section{Supramolecular features}

In the crystal, molecules are linked by weak $\mathrm{C}-\mathrm{H} \cdots \mathrm{O}$ hydrogen bonds (Table 1), forming chains propagating along [010] (Fig. 2). In addition, $\pi-\pi$ stacking interactions with centroid-centroid distances $C g 1 \cdots C g 1(2-x,-y, 1-z)$ of 3.902 (2) $\AA$ and $C g 1 \cdots C g 2(1-x,-y, 1-z)$ of $3.908(2) \AA$ where $C g 1$ and $C g 2$ are the centroids of the $\mathrm{C} 1 / \mathrm{C} 5 / \mathrm{C} 6 / \mathrm{C} 10$ $\mathrm{C} 12$ and $\mathrm{O} 2 / \mathrm{C} 6-\mathrm{C} 10$ rings, respectively, link the hydrogenbonded chains, forming layers parallel (001) (Fig. 3).

\section{Database survey}

A search of the Cambridge Structural Database (CSD, Version 5.38, update November, 2016; Groom et al., 2016) gave more than thirty five hits for both linear and angular pyranocoumarin (psoralen class) structures. They include closely related structures [CSD refcodes AMYROL (Kato, 1970), FUGVOS (Thailambal \& Pattabhi, 1987), AMYROL01 (Bauri et al., 2006, 2017)] and a number of structures with various substituents at $\mathrm{C} 3$ and $\mathrm{C} 4$, many of which are natural products.

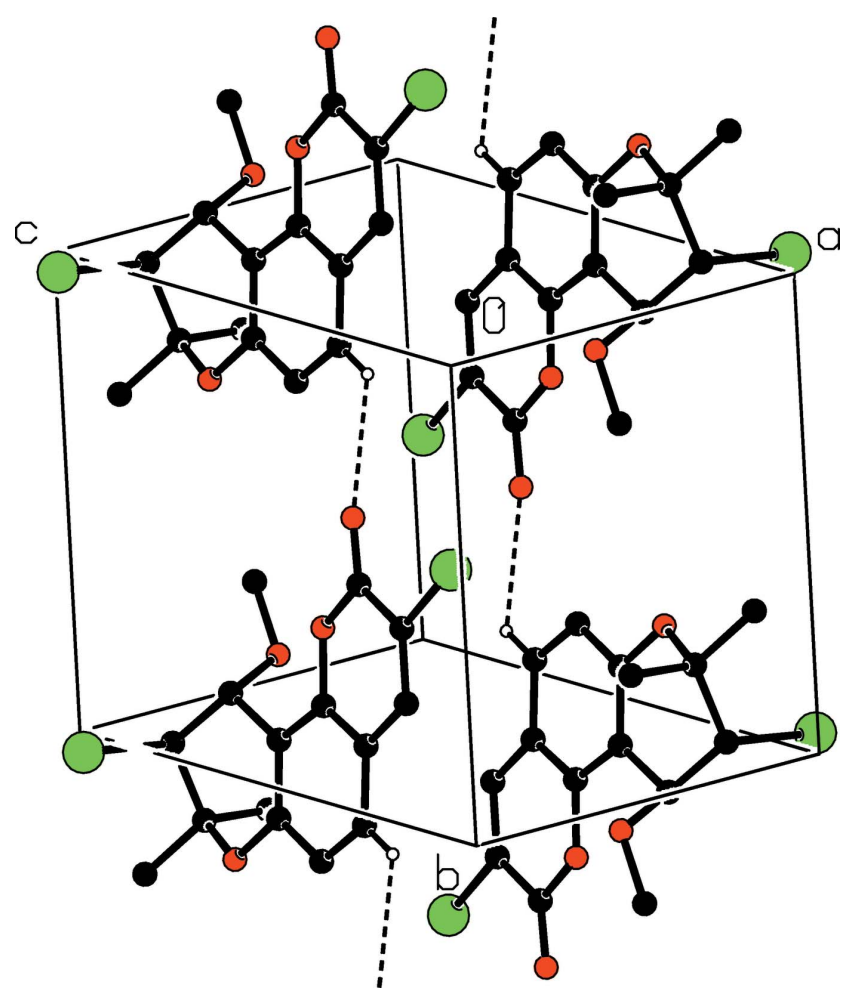

Figure 2

Part of the crystal structure with weak $\mathrm{C}-\mathrm{H} \cdots \mathrm{O}$ hydrogen bonds shown as dashed lines. Only the $\mathrm{H}$ atoms involved in hydrogen bonds are shown. 
Table 2

Experimental details.

\begin{tabular}{|c|c|}
\hline \multicolumn{2}{|l|}{ Crystal data } \\
\hline Chemical formula & $\mathrm{C}_{15} \mathrm{H}_{14} \mathrm{Br}_{2} \mathrm{O}_{4}$ \\
\hline$M_{\mathrm{r}}$ & 418.08 \\
\hline Crystal system, space group & Triclinic, $P \overline{1}$ \\
\hline Temperature $(\mathrm{K})$ & 299 \\
\hline$a, b, c(\AA)$ & $7.119(1), 8.519(1), 13.366$ (2) \\
\hline$\alpha, \beta, \gamma\left({ }^{\circ}\right)$ & $105.34(2), 90.45(1), 103.38(2)$ \\
\hline$V\left(\AA^{3}\right)$ & $758.4(2)$ \\
\hline$Z$ & 2 \\
\hline Radiation type & Mo $K \alpha$ \\
\hline$\mu\left(\mathrm{mm}^{-1}\right)$ & 5.36 \\
\hline Crystal size (mm) & $0.20 \times 0.20 \times 0.16$ \\
\hline \multicolumn{2}{|l|}{ Data collection } \\
\hline Diffractometer & $\begin{array}{l}\text { Oxford Diffraction Xcalibur } \\
\text { single-crystal X-ray } \\
\text { diffractometer with a Sapphire } \\
\text { CCD detector }\end{array}$ \\
\hline Absorption correction & $\begin{array}{l}\text { Multi-scan (CrysAlis RED; Oxford } \\
\text { Diffraction, 2009) }\end{array}$ \\
\hline$T_{\min }, T_{\max }$ & $0.364,0.423$ \\
\hline $\begin{array}{l}\text { No. of measured, independent and } \\
\text { observed }[I>2 \sigma(I)] \text { reflections }\end{array}$ & $5172,2764,2144$ \\
\hline$R_{\text {int }}$ & 0.015 \\
\hline$(\sin \theta / \lambda)_{\max }\left(\AA^{-1}\right)$ & 0.602 \\
\hline \multicolumn{2}{|l|}{ Refinement } \\
\hline$R\left[F^{2}>2 \sigma\left(F^{2}\right)\right], w R\left(F^{2}\right), S$ & $0.035,0.116,0.85$ \\
\hline No. of reflections & 2764 \\
\hline No. of parameters & 193 \\
\hline $\mathrm{H}$-atom treatment & $\mathrm{H}$-atom parameters constrained \\
\hline$\Delta \rho_{\max }, \Delta \rho_{\min }\left(\mathrm{e} \AA^{-3}\right)$ & $0.46,-0.42$ \\
\hline
\end{tabular}

Computer programs: CrysAlis CCD and CrysAlis RED (Oxford Diffraction, 2009), SHELXS97 and SHELXL97 (Sheldrick, 2008) and PLATON (Spek, 2009).

\section{Synthesis and crystallization}

The title compound is a colourless solid substance formed on bromination of the naturally occurring seseline isolated from the methanol extract of T. stictocarpum by means of column chromatography over $\mathrm{SiO}_{2}$ gel with gradient elution by using a mixture of the binary solvents hexane and ethyl acetate. The bromination was conducted using NBS in methanol at room temperature with continuous stirring by means of mechanical stirrer over a period of $12 \mathrm{~h}$. The reaction product was worked up by the usual method to yield crude product, which was then purified by solvent elution to yield the title compound. A colourless prism-shaped crystal was obtained after recrystallization $(\times 3)$ from ethyl acetate:hexane (1:4) at room temperature by slow evaporation of the solvents. NMR analysis: ${ }^{1} \mathrm{H} \mathrm{NMR}$ data $\left(\mathrm{CDCl}_{3}, 200 \mathrm{MHz}\right): \delta_{\mathrm{H}} 8.02(s, 1 \mathrm{H}, \mathrm{H}-$ 9), $7.32(d, 1 \mathrm{H}, J=8.80 \mathrm{~Hz}, \mathrm{H}-12), 6.82(d, 1 \mathrm{H}, J=8.80 \mathrm{~Hz}, \mathrm{H}-$ 11), $5.36(d, 1 \mathrm{H}, J=6.8 \mathrm{~Hz}, \mathrm{H}-4), 4.26(d, 1 \mathrm{H}, J=6.8 \mathrm{~Hz}, \mathrm{H}-3)$, $3.56\left(s, 3 \mathrm{H},-\mathrm{OCH}_{3}, \mathrm{H}-13\right), 1.50\left(s, 3 \mathrm{H}, \mathrm{CH}_{3}, \mathrm{H}-13\right), 1.54(s, 3 \mathrm{H}$, $\left.\mathrm{CH}_{3}, \mathrm{H}-14\right)$.

\section{Refinement}

Crystal data, data collection and structure refinement details are summarized in Table 2. $\mathrm{H}$ atoms were included in calcu-

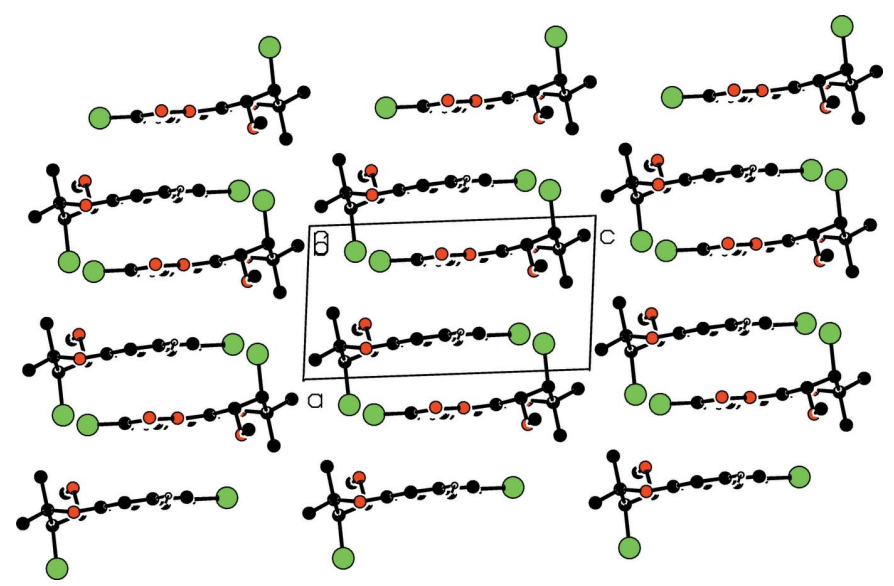

Figure 3

Part of the crystal structure showing layers of molecules parallel to (001).

lated positions and treated as riding atoms with $\mathrm{C}-\mathrm{H}=0.93-$ $0.98 \AA$ with $\mathrm{U}_{\text {iso }}(\mathrm{H})=1.2 U_{\text {eq }}(\mathrm{C})$.

\section{Acknowledgements}

The authors thank Professor Dr Hartmut, FG Strukturforschung, Material-und Geowissenschaften, Technische Universität Darmstadt, for his kind cooperation with the data collection and providing diffractometer time.

\section{References}

Appendino, G., Bianchi, F., Bader, A., Campagnuolo, C., Fattorusso, E., Taglialatela-Scafati, O., Blanco-Molina, M., Macho, A., Fiebich, B. L., Bremner, P., Heinrich, M., Ballero, M. \& Muñoz, E. (2004). J. Nat. Prod. 67, 532-536.

Banerjee, S., Ghosh, B. K., Bauri, A. K. \& Bhattacharya, S. (2014). J Spectrosc Dyn, 4, 29-34.

Bauri, A. K., Foro, S., Lindner, H.-J. \& Nayak, S. K. (2006). Acta Cryst. E62, o1340-o1341.

Bauri, A. K., Foro, S. \& Rahman, A. F. M. M. (2017). Acta Cryst. E73, 453-455.

Chen, Y., Fan, G., Zhang, Q., Wu, H. \& Wu, Y. (2007). J. Pharm. Biomed. Anal. 43, 926-936.

Chen, D., Wang, J., Jiang, Y., Zhou, T., Fan, G. \& Wu, Y. (2009). J. Pharm. Biomed. Anal. 50, 695-702.

Conconi, M. T., Montesi, F. \& Parnigotto, P. P. (1998). Basic Clin. Pharmacol. Toxicol. 82, 193-198.

Conforti, F., Marrelli, M., Menichini, F., Bonesi, M., Statti, G., Provenzano, E. \& Menichini, F. (2009). Current Drug Ther. 4, 3858.

Ghosh, B. K., Bauri, A. K., Bhattacharya, S. \& Banerjee, S. (2014). Spectrochim. Acta Part A, 125, 90-98.

Groom, C. R., Bruno, I. J., Lightfoot, M. P. \& Ward, S. C. (2016). Acta Cryst. B72, 171-179.

Kato, K. (1970). Acta Cryst. B26, 2022-2029.

March, K. L., Patton, B. L., Wilensky, R. L. \& Hathaway, D. R. (1993). Circulation, 87, 184-191.

Oxford Diffraction (2009). CrysAlis CCD and CrysAlis RED. Oxford Diffraction Ltd, Abingdon, England.

Sheldrick, G. M. (2008). Acta Cryst. A64, 112-122.

Spek, A. L. (2009). Acta Cryst. D65, 148-155.

Thailambal, V. G. \& Pattabhi, V. (1987). Acta Cryst. C43, 2369-2372. 


\section{supporting information}

Acta Cryst. (2017). E73, 774-776 [https://doi.org/10.1107/S2056989017006132]

\section{Crystal structure of dibromomethoxyseselin (DBMS), a photobiologically active pyranocoumarin}

\section{A. K. Bauri, Sabine Foro and A. F. M. M. Rahman}

Computing details

Data collection: CrysAlis CCD (Oxford Diffraction, 2009); cell refinement: CrysAlis RED (Oxford Diffraction, 2009); data reduction: CrysAlis RED (Oxford Diffraction, 2009); program(s) used to solve structure: SHELXS97 (Sheldrick, 2008); program(s) used to refine structure: SHELXL97 (Sheldrick, 2008); molecular graphics: PLATON (Spek, 2009); software used to prepare material for publication: SHELXL97 (Sheldrick, 2008).

rac-(9S,10R)-3,9-Dibromo-10-methoxy-8,8-dimethyl-9,10-dihydropyrano[2,3-h]chromen-2(8H)-one

\section{Crystal data}

$\mathrm{C}_{15} \mathrm{H}_{14} \mathrm{Br}_{2} \mathrm{O}_{4}$

$M_{r}=418.08$

Triclinic, $P \overline{1}$

Hall symbol: -P 1

$a=7.119$ (1) $\AA$

$b=8.519(1) \AA$

$c=13.366(2) \AA$

$\alpha=105.34(2)^{\circ}$

$\beta=90.45(1)^{\circ}$

$\gamma=103.38(2)^{\circ}$

$V=758.4(2) \AA^{3}$

Data collection

Oxford Diffraction Xcalibur single-crystal Xray diffractometer with a Sapphire CCD detector

Radiation source: fine-focus sealed tube Graphite monochromator

Rotation method data acquisition using $\omega$ and phi scans.

Absorption correction: multi-scan

(CrysAlis RED; Oxford Diffraction, 2009)

Refinement

Refinement on $F^{2}$

Least-squares matrix: full

$R\left[F^{2}>2 \sigma\left(F^{2}\right)\right]=0.035$

$w R\left(F^{2}\right)=0.116$

$S=0.85$

2764 reflections

193 parameters
$Z=2$

$F(000)=412$

$D_{\mathrm{x}}=1.831 \mathrm{Mg} \mathrm{m}^{-3}$

Mo $K \alpha$ radiation, $\lambda=0.71073 \AA$

Cell parameters from 2165 reflections

$\theta=2.6-27.9^{\circ}$

$\mu=5.36 \mathrm{~mm}^{-1}$

$T=299 \mathrm{~K}$

Prism, colourless

$0.20 \times 0.20 \times 0.16 \mathrm{~mm}$

$T_{\min }=0.364, T_{\max }=0.423$

5172 measured reflections

2764 independent reflections

2144 reflections with $I>2 \sigma(I)$

$R_{\text {int }}=0.015$

$\theta_{\max }=25.4^{\circ}, \theta_{\min }=2.6^{\circ}$

$h=-8 \rightarrow 8$

$k=-8 \rightarrow 10$

$l=-16 \rightarrow 12$

0 restraints

Primary atom site location: structure-invariant direct methods

Secondary atom site location: difference Fourier map

Hydrogen site location: inferred from neighbouring sites 
H-atom parameters constrained

$w=1 /\left[\sigma^{2}\left(F_{\mathrm{o}}^{2}\right)+(0.1 P)^{2}\right]$

where $P=\left(F_{\mathrm{o}}^{2}+2 F_{\mathrm{c}}^{2}\right) / 3$

$$
\begin{aligned}
& (\Delta / \sigma)_{\max }=0.004 \\
& \Delta \rho_{\max }=0.46 \mathrm{e}^{-3} \\
& \Delta \rho_{\min }=-0.42 \mathrm{e} \AA^{-3}
\end{aligned}
$$

Special details

Geometry. All esds (except the esd in the dihedral angle between two 1.s. planes) are estimated using the full covariance matrix. The cell esds are taken into account individually in the estimation of esds in distances, angles and torsion angles; correlations between esds in cell parameters are only used when they are defined by crystal symmetry. An approximate (isotropic) treatment of cell esds is used for estimating esds involving l.s. planes.

Refinement. Refinement of $\mathrm{F}^{2}$ against ALL reflections. The weighted R-factor $\mathrm{wR}$ and goodness of fit $\mathrm{S}$ are based on $\mathrm{F}^{2}$, conventional R-factors $\mathrm{R}$ are based on $\mathrm{F}$, with $\mathrm{F}$ set to zero for negative $\mathrm{F}^{2}$. The threshold expression of $\mathrm{F}^{2}$ \&gt;

$2 \operatorname{sigma}\left(\mathrm{F}^{2}\right)$ is used only for calculating R-factors (gt) etc. and is not relevant to the choice of reflections for refinement. Rfactors based on $\mathrm{F}^{2}$ are statistically about twice as large as those based on F, and R-factors based on ALL data will be

\begin{tabular}{|c|c|c|c|c|}
\hline & $x$ & $y$ & $z$ & $U_{\text {iso }} * / U_{\text {eq }}$ \\
\hline Br1 & $1.17651(6)$ & $0.02924(6)$ & $0.16407(4)$ & $0.04731(18)$ \\
\hline $\mathrm{Br} 2$ & $0.74960(8)$ & $0.46658(6)$ & $0.74871(4)$ & $0.0602(2)$ \\
\hline O1 & $0.8143(4)$ & -0.2513 & $0.2144(2)$ & $0.0405(7)$ \\
\hline $\mathrm{O} 2$ & $0.7746(4)$ & 0.2766 & $0.4357(2)$ & $0.0399(7)$ \\
\hline $\mathrm{O} 3$ & $0.6525(4)$ & 0.1428 & $0.2086(2)$ & $0.0416(7)$ \\
\hline $\mathrm{O} 4$ & $0.7816(6)$ & $0.5300(4)$ & $0.5337(3)$ & $0.0694(11)$ \\
\hline $\mathrm{C} 1$ & $0.7877(5)$ & $-0.1487(5)$ & 0.3085 & $0.0322(8)$ \\
\hline $\mathrm{C} 2$ & $0.7930(6)$ & $-0.1956(5)$ & $0.1231(3)$ & $0.0407(9)$ \\
\hline $\mathrm{C} 3$ & $0.8945(5)$ & $-0.0109(5)$ & 0.1422 & $0.0345(8)$ \\
\hline H3 & 0.8663 & 0.0240 & 0.0807 & $0.041^{*}$ \\
\hline $\mathrm{C} 4$ & $0.8264(5)$ & $0.1028(5)$ & 0.2368 & $0.0319(8)$ \\
\hline $\mathrm{H} 4$ & 0.9274 & 0.2070 & 0.2619 & $0.038 *$ \\
\hline $\mathrm{C} 5$ & $0.7908(5)$ & $0.0188(5)$ & $0.3224(3)$ & $0.0305(8)$ \\
\hline C6 & $0.7703(5)$ & $0.1103(4)$ & $0.4232(3)$ & $0.0299(8)$ \\
\hline $\mathrm{C} 7$ & $0.7715(6)$ & $0.3884(5)$ & 0.5309 & $0.0437(10)$ \\
\hline $\mathrm{C} 8$ & $0.7530(6)$ & $0.3147(5)$ & $0.6187(3)$ & $0.0357(9)$ \\
\hline C9 & $0.7392(5)$ & $0.1528(5)$ & 0.6078 & $0.0352(9)$ \\
\hline $\mathrm{H} 9$ & 0.7232 & 0.1108 & 0.6656 & $0.042 *$ \\
\hline $\mathrm{C} 10$ & $0.7488(5)$ & $0.0428(5)$ & $0.5074(3)$ & $0.0305(8)$ \\
\hline $\mathrm{C} 11$ & $0.7447(5)$ & $-0.1286(5)$ & $0.4888(3)$ & $0.0345(9)$ \\
\hline H11 & 0.7290 & -0.1781 & 0.5435 & $0.041 *$ \\
\hline $\mathrm{C} 12$ & $0.7636(6)$ & $-0.2230(5)$ & 0.3909 & $0.0355(9)$ \\
\hline H12 & 0.7605 & -0.3362 & 0.3790 & $0.043 *$ \\
\hline $\mathrm{C} 13$ & $0.5762(7)$ & $-0.2223(6)$ & $0.0942(4)$ & $0.0553(12)$ \\
\hline H13A & 0.5609 & -0.1819 & 0.0348 & $0.066^{*}$ \\
\hline H13B & 0.5182 & -0.1619 & 0.1518 & $0.066^{*}$ \\
\hline $\mathrm{H} 13 \mathrm{C}$ & 0.5142 & -0.3397 & 0.0780 & $0.066^{*}$ \\
\hline $\mathrm{C} 14$ & $0.8775(8)$ & $-0.3121(6)$ & $0.0381(4)$ & 0.0590 (13) \\
\hline $\mathrm{H} 14 \mathrm{~A}$ & 1.0133 & -0.2949 & 0.0553 & $0.071 *$ \\
\hline H14B & 0.8597 & -0.2880 & -0.0270 & $0.071 *$ \\
\hline $\mathrm{H} 14 \mathrm{C}$ & 0.8128 & -0.4265 & 0.0325 & $0.071 *$ \\
\hline
\end{tabular}
even larger.

Fractional atomic coordinates and isotropic or equivalent isotropic displacement parameters $\left(\hat{A}^{2}\right)$ 


\begin{tabular}{lllll} 
C15 & $0.6827(8)$ & $0.2953(7)$ & $0.1849(5)$ & $0.0697(16)$ \\
H15A & 0.7553 & 0.3827 & 0.2421 & $0.084^{*}$ \\
H15B & 0.5602 & 0.3182 & 0.1723 & $0.084^{*}$ \\
H15C & 0.7537 & 0.2904 & 0.1238 & $0.084^{*}$ \\
\hline
\end{tabular}

Atomic displacement parameters $\left(\AA^{2}\right)$

\begin{tabular}{lllllll}
\hline & $U^{11}$ & $U^{22}$ & $U^{33}$ & $U^{12}$ & $U^{13}$ & $U^{23}$ \\
\hline Br1 & $0.0397(3)$ & $0.0566(3)$ & $0.0543(3)$ & $0.0166(2)$ & $0.0127(2)$ & $0.0253(2)$ \\
Br2 & $0.0801(4)$ & $0.0457(3)$ & $0.0433(3)$ & $0.0097(2)$ & $0.0166(2)$ & $-0.0031(2)$ \\
O1 & $0.0586(18)$ & $0.0296(14)$ & $0.0347(15)$ & $0.0149(13)$ & $0.0110(13)$ & $0.0074(12)$ \\
O2 & $0.0640(19)$ & $0.0262(14)$ & $0.0344(15)$ & $0.0166(13)$ & $0.0096(13)$ & $0.0114(11)$ \\
O3 & $0.0400(15)$ & $0.0435(17)$ & $0.0481(17)$ & $0.0152(13)$ & $0.0043(13)$ & $0.0197(14)$ \\
O4 & $0.127(3)$ & $0.0293(18)$ & $0.060(2)$ & $0.0305(19)$ & $0.021(2)$ & $0.0158(15)$ \\
C1 & $0.0314(19)$ & $0.031(2)$ & $0.036(2)$ & $0.0078(16)$ & $0.0052(16)$ & $0.0104(17)$ \\
C2 & $0.051(2)$ & $0.039(2)$ & $0.031(2)$ & $0.0126(19)$ & $0.0068(18)$ & $0.0062(17)$ \\
C3 & $0.036(2)$ & $0.040(2)$ & $0.032(2)$ & $0.0129(17)$ & $0.0100(16)$ & $0.0139(17)$ \\
C4 & $0.035(2)$ & $0.031(2)$ & $0.033(2)$ & $0.0101(16)$ & $0.0032(16)$ & $0.0112(16)$ \\
C5 & $0.0319(19)$ & $0.0293(19)$ & $0.033(2)$ & $0.0083(16)$ & $0.0065(15)$ & $0.0120(16)$ \\
C6 & $0.0313(19)$ & $0.0245(19)$ & $0.035(2)$ & $0.0061(15)$ & $0.0039(16)$ & $0.0096(15)$ \\
C7 & $0.051(3)$ & $0.038(3)$ & $0.043(2)$ & $0.016(2)$ & $0.008(2)$ & $0.0089(19)$ \\
C8 & $0.038(2)$ & $0.034(2)$ & $0.034(2)$ & $0.0105(17)$ & $0.0062(17)$ & $0.0061(16)$ \\
C9 & $0.035(2)$ & $0.040(2)$ & $0.032(2)$ & $0.0077(17)$ & $0.0048(16)$ & $0.0122(17)$ \\
C10 & $0.0287(18)$ & $0.032(2)$ & $0.032(2)$ & $0.0075(16)$ & $0.0067(15)$ & $0.0103(16)$ \\
C11 & $0.036(2)$ & $0.033(2)$ & $0.039(2)$ & $0.0070(17)$ & $0.0029(17)$ & $0.0181(17)$ \\
C12 & $0.042(2)$ & $0.027(2)$ & $0.041(2)$ & $0.0099(17)$ & $0.0039(18)$ & $0.0131(17)$ \\
C13 & $0.055(3)$ & $0.050(3)$ & $0.048(3)$ & $-0.005(2)$ & $-0.001(2)$ & $0.008(2)$ \\
C14 & $0.088(4)$ & $0.041(3)$ & $0.044(3)$ & $0.019(2)$ & $0.020(3)$ & $0.002(2)$ \\
C15 & $0.068(4)$ & $0.065(4)$ & $0.091(4)$ & $0.029(3)$ & $-0.003(3)$ & $0.035(3)$ \\
& & & & & & \\
\hline
\end{tabular}

Geometric parameters $\left(\AA,{ }^{o}\right)$

\begin{tabular}{llll}
\hline $\mathrm{Br} 1-\mathrm{C} 3$ & $1.963(4)$ & $\mathrm{C} 6-\mathrm{C} 10$ & $1.388(5)$ \\
$\mathrm{Br} 2-\mathrm{C} 8$ & $1.876(4)$ & $\mathrm{C} 7-\mathrm{C} 8$ & $1.463(6)$ \\
$\mathrm{O} 1-\mathrm{C} 1$ & $1.371(4)$ & $\mathrm{C} 8-\mathrm{C} 9$ & $1.328(5)$ \\
$\mathrm{O} 1-\mathrm{C} 2$ & $1.440(5)$ & $\mathrm{C} 9-\mathrm{C} 10$ & $1.432(5)$ \\
$\mathrm{O} 2-\mathrm{C} 6$ & $1.375(4)$ & $\mathrm{C} 9-\mathrm{H} 9$ & 0.9300 \\
$\mathrm{O} 2-\mathrm{C} 7$ & $1.377(5)$ & $\mathrm{C} 10-\mathrm{C} 11$ & $1.408(5)$ \\
$\mathrm{O} 3-\mathrm{C} 15$ & $1.386(5)$ & $\mathrm{C} 11-\mathrm{C} 12$ & $1.369(5)$ \\
$\mathrm{O} 3-\mathrm{C} 4$ & $1.431(4)$ & $\mathrm{C} 11-\mathrm{H} 11$ & 0.9300 \\
$\mathrm{O} 4-\mathrm{C} 7$ & $1.183(5)$ & $\mathrm{C} 12-\mathrm{H} 12$ & 0.9300 \\
$\mathrm{C} 1-\mathrm{C} 5$ & $1.384(5)$ & $\mathrm{C} 13-\mathrm{H} 13 \mathrm{~A}$ & 0.9600 \\
$\mathrm{C} 1-\mathrm{C} 12$ & $1.401(5)$ & $\mathrm{C} 13-\mathrm{H} 13 \mathrm{~B}$ & 0.9600 \\
$\mathrm{C} 2-\mathrm{C} 3$ & $1.524(6)$ & $\mathrm{C} 13-\mathrm{H} 13 \mathrm{C}$ & 0.9600 \\
$\mathrm{C} 2-\mathrm{C} 14$ & $1.526(5)$ & $\mathrm{C} 14-\mathrm{H} 14 \mathrm{~A}$ & 0.9600 \\
$\mathrm{C} 2-\mathrm{C} 13$ & $1.538(6)$ & $\mathrm{C} 14-\mathrm{H} 14 \mathrm{~B}$ & 0.9600 \\
$\mathrm{C} 3-\mathrm{C} 4$ & $1.533(5)$ & $\mathrm{C} 14-\mathrm{H} 14 \mathrm{C}$ & 0.9600 \\
$\mathrm{C} 3-\mathrm{H} 3$ & 0.9800 & $\mathrm{C} 15-\mathrm{H} 15 \mathrm{~A}$ & 0.9600
\end{tabular}




\begin{tabular}{|c|c|c|c|}
\hline $\mathrm{C} 4-\mathrm{C} 5$ & $1.496(5)$ & C15-H15B & 0.9600 \\
\hline $\mathrm{C} 4-\mathrm{H} 4$ & 0.9800 & $\mathrm{C} 15-\mathrm{H} 15 \mathrm{C}$ & 0.9600 \\
\hline $\mathrm{C} 5-\mathrm{C} 6$ & $1.394(5)$ & & \\
\hline $\mathrm{C} 1-\mathrm{O} 1-\mathrm{C} 2$ & $117.6(3)$ & $\mathrm{C} 9-\mathrm{C} 8-\mathrm{C} 7$ & $122.9(4)$ \\
\hline $\mathrm{C} 6-\mathrm{O} 2-\mathrm{C} 7$ & $123.4(3)$ & $\mathrm{C} 9-\mathrm{C} 8-\mathrm{Br} 2$ & $122.2(3)$ \\
\hline $\mathrm{C} 15-\mathrm{O} 3-\mathrm{C} 4$ & $114.0(3)$ & $\mathrm{C} 7-\mathrm{C} 8-\mathrm{Br} 2$ & $114.9(3)$ \\
\hline $\mathrm{O} 1-\mathrm{C} 1-\mathrm{C} 5$ & $122.4(3)$ & $\mathrm{C} 8-\mathrm{C} 9-\mathrm{C} 10$ & $120.1(4)$ \\
\hline $\mathrm{O} 1-\mathrm{C} 1-\mathrm{C} 12$ & $115.6(3)$ & $\mathrm{C} 8-\mathrm{C} 9-\mathrm{H} 9$ & 119.9 \\
\hline $\mathrm{C} 5-\mathrm{C} 1-\mathrm{C} 12$ & $122.0(3)$ & $\mathrm{C} 10-\mathrm{C} 9-\mathrm{H} 9$ & 119.9 \\
\hline $\mathrm{O} 1-\mathrm{C} 2-\mathrm{C} 3$ & $111.0(3)$ & $\mathrm{C} 6-\mathrm{C} 10-\mathrm{C} 11$ & $117.6(3)$ \\
\hline $\mathrm{O} 1-\mathrm{C} 2-\mathrm{C} 14$ & $104.5(3)$ & $\mathrm{C} 6-\mathrm{C} 10-\mathrm{C} 9$ & $118.1(3)$ \\
\hline $\mathrm{C} 3-\mathrm{C} 2-\mathrm{C} 14$ & $113.4(3)$ & $\mathrm{C} 11-\mathrm{C} 10-\mathrm{C} 9$ & $124.3(3)$ \\
\hline $\mathrm{O} 1-\mathrm{C} 2-\mathrm{C} 13$ & $109.0(3)$ & $\mathrm{C} 12-\mathrm{C} 11-\mathrm{C} 10$ & $120.4(3)$ \\
\hline $\mathrm{C} 3-\mathrm{C} 2-\mathrm{C} 13$ & $109.7(3)$ & $\mathrm{C} 12-\mathrm{C} 11-\mathrm{H} 11$ & 119.8 \\
\hline $\mathrm{C} 14-\mathrm{C} 2-\mathrm{C} 13$ & $109.1(4)$ & $\mathrm{C} 10-\mathrm{C} 11-\mathrm{H} 11$ & 119.8 \\
\hline $\mathrm{C} 2-\mathrm{C} 3-\mathrm{C} 4$ & $113.0(3)$ & $\mathrm{C} 11-\mathrm{C} 12-\mathrm{C} 1$ & $119.9(3)$ \\
\hline $\mathrm{C} 2-\mathrm{C} 3-\mathrm{Br} 1$ & $112.1(3)$ & $\mathrm{C} 11-\mathrm{C} 12-\mathrm{H} 12$ & 120.0 \\
\hline $\mathrm{C} 4-\mathrm{C} 3-\mathrm{Br} 1$ & $107.3(3)$ & $\mathrm{C} 1-\mathrm{C} 12-\mathrm{H} 12$ & 120.0 \\
\hline $\mathrm{C} 2-\mathrm{C} 3-\mathrm{H} 3$ & 108.1 & $\mathrm{C} 2-\mathrm{C} 13-\mathrm{H} 13 \mathrm{~A}$ & 109.5 \\
\hline $\mathrm{C} 4-\mathrm{C} 3-\mathrm{H} 3$ & 108.1 & $\mathrm{C} 2-\mathrm{C} 13-\mathrm{H} 13 \mathrm{~B}$ & 109.5 \\
\hline $\mathrm{Br} 1-\mathrm{C} 3-\mathrm{H} 3$ & 108.1 & $\mathrm{H} 13 \mathrm{~A}-\mathrm{C} 13-\mathrm{H} 13 \mathrm{~B}$ & 109.5 \\
\hline $\mathrm{O} 3-\mathrm{C} 4-\mathrm{C} 5$ & $109.4(3)$ & $\mathrm{C} 2-\mathrm{C} 13-\mathrm{H} 13 \mathrm{C}$ & 109.5 \\
\hline $\mathrm{O} 3-\mathrm{C} 4-\mathrm{C} 3$ & $110.3(3)$ & $\mathrm{H} 13 \mathrm{~A}-\mathrm{C} 13-\mathrm{H} 13 \mathrm{C}$ & 109.5 \\
\hline $\mathrm{C} 5-\mathrm{C} 4-\mathrm{C} 3$ & $110.5(3)$ & $\mathrm{H} 13 \mathrm{~B}-\mathrm{C} 13-\mathrm{H} 13 \mathrm{C}$ & 109.5 \\
\hline $\mathrm{O} 3-\mathrm{C} 4-\mathrm{H} 4$ & 108.8 & $\mathrm{C} 2-\mathrm{C} 14-\mathrm{H} 14 \mathrm{~A}$ & 109.5 \\
\hline $\mathrm{C} 5-\mathrm{C} 4-\mathrm{H} 4$ & 108.8 & $\mathrm{C} 2-\mathrm{C} 14-\mathrm{H} 14 \mathrm{~B}$ & 109.5 \\
\hline $\mathrm{C} 3-\mathrm{C} 4-\mathrm{H} 4$ & 108.8 & $\mathrm{H} 14 \mathrm{~A}-\mathrm{C} 14-\mathrm{H} 14 \mathrm{~B}$ & 109.5 \\
\hline $\mathrm{C} 1-\mathrm{C} 5-\mathrm{C} 6$ & $116.3(3)$ & $\mathrm{C} 2-\mathrm{C} 14-\mathrm{H} 14 \mathrm{C}$ & 109.5 \\
\hline $\mathrm{C} 1-\mathrm{C} 5-\mathrm{C} 4$ & $122.9(3)$ & $\mathrm{H} 14 \mathrm{~A}-\mathrm{C} 14-\mathrm{H} 14 \mathrm{C}$ & 109.5 \\
\hline $\mathrm{C} 6-\mathrm{C} 5-\mathrm{C} 4$ & $120.7(3)$ & $\mathrm{H} 14 \mathrm{~B}-\mathrm{C} 14-\mathrm{H} 14 \mathrm{C}$ & 109.5 \\
\hline $\mathrm{O} 2-\mathrm{C} 6-\mathrm{C} 10$ & $120.6(3)$ & $\mathrm{O} 3-\mathrm{C} 15-\mathrm{H} 15 \mathrm{~A}$ & 109.5 \\
\hline $\mathrm{O} 2-\mathrm{C} 6-\mathrm{C} 5$ & $115.6(3)$ & $\mathrm{O} 3-\mathrm{C} 15-\mathrm{H} 15 \mathrm{~B}$ & 109.5 \\
\hline $\mathrm{C} 10-\mathrm{C} 6-\mathrm{C} 5$ & $123.8(3)$ & $\mathrm{H} 15 \mathrm{~A}-\mathrm{C} 15-\mathrm{H} 15 \mathrm{~B}$ & 109.5 \\
\hline $\mathrm{O} 4-\mathrm{C} 7-\mathrm{O} 2$ & $118.2(4)$ & $\mathrm{O} 3-\mathrm{C} 15-\mathrm{H} 15 \mathrm{C}$ & 109.5 \\
\hline $\mathrm{O} 4-\mathrm{C} 7-\mathrm{C} 8$ & $127.1(4)$ & $\mathrm{H} 15 \mathrm{~A}-\mathrm{C} 15-\mathrm{H} 15 \mathrm{C}$ & 109.5 \\
\hline $\mathrm{O} 2-\mathrm{C} 7-\mathrm{C} 8$ & $114.7(3)$ & $\mathrm{H} 15 \mathrm{~B}-\mathrm{C} 15-\mathrm{H} 15 \mathrm{C}$ & 109.5 \\
\hline $\mathrm{C} 2-\mathrm{O} 1-\mathrm{C} 1-\mathrm{C} 5$ & $-16.8(6)$ & $\mathrm{C} 7-\mathrm{O} 2-\mathrm{C} 6-\mathrm{C} 10$ & $4.5(6)$ \\
\hline $\mathrm{C} 2-\mathrm{O} 1-\mathrm{C} 1-\mathrm{C} 12$ & $165.3(3)$ & $\mathrm{C} 7-\mathrm{O} 2-\mathrm{C} 6-\mathrm{C} 5$ & $-174.8(3)$ \\
\hline $\mathrm{C} 1-\mathrm{O} 1-\mathrm{C} 2-\mathrm{C} 3$ & $44.2(5)$ & $\mathrm{C} 1-\mathrm{C} 5-\mathrm{C} 6-\mathrm{O} 2$ & $179.7(3)$ \\
\hline $\mathrm{C} 1-\mathrm{O} 1-\mathrm{C} 2-\mathrm{C} 14$ & $166.8(3)$ & $\mathrm{C} 4-\mathrm{C} 5-\mathrm{C} 6-\mathrm{O} 2$ & $3.4(5)$ \\
\hline $\mathrm{C} 1-\mathrm{O} 1-\mathrm{C} 2-\mathrm{C} 13$ & $-76.7(4)$ & $\mathrm{C} 1-\mathrm{C} 5-\mathrm{C} 6-\mathrm{C} 10$ & $0.4(6)$ \\
\hline $\mathrm{O} 1-\mathrm{C} 2-\mathrm{C} 3-\mathrm{C} 4$ & $-55.3(4)$ & $\mathrm{C} 4-\mathrm{C} 5-\mathrm{C} 6-\mathrm{C} 10$ & $-176.0(3)$ \\
\hline $\mathrm{C} 14-\mathrm{C} 2-\mathrm{C} 3-\mathrm{C} 4$ & $-172.6(4)$ & $\mathrm{C} 6-\mathrm{O} 2-\mathrm{C} 7-\mathrm{O} 4$ & $177.8(4)$ \\
\hline $\mathrm{C} 13-\mathrm{C} 2-\mathrm{C} 3-\mathrm{C} 4$ & $65.1(4)$ & $\mathrm{C} 6-\mathrm{O} 2-\mathrm{C} 7-\mathrm{C} 8$ & $-3.0(6)$ \\
\hline $\mathrm{O} 1-\mathrm{C} 2-\mathrm{C} 3-\mathrm{Br} 1$ & $66.0(3)$ & $\mathrm{O} 4-\mathrm{C} 7-\mathrm{C} 8-\mathrm{C} 9$ & $178.8(5)$ \\
\hline $\mathrm{C} 14-\mathrm{C} 2-\mathrm{C} 3-\mathrm{Br} 1$ & $-51.3(4)$ & $\mathrm{O} 2-\mathrm{C} 7-\mathrm{C} 8-\mathrm{C} 9$ & $-0.3(6)$ \\
\hline
\end{tabular}




$\begin{array}{llll}\mathrm{C} 13-\mathrm{C} 2-\mathrm{C} 3-\mathrm{Br} 1 & -173.5(3) & \mathrm{O} 4-\mathrm{C} 7-\mathrm{C} 8-\mathrm{Br} 2 & -1.1(7) \\ \mathrm{C} 15-\mathrm{O} 3-\mathrm{C} 4-\mathrm{C} 5 & 142.1(4) & \mathrm{O} 2-\mathrm{C} 7-\mathrm{C} 8-\mathrm{Br} 2 & 179.8(3) \\ \mathrm{C} 15-\mathrm{O} 3-\mathrm{C} 4-\mathrm{C} 3 & -96.1(4) & \mathrm{C} 7-\mathrm{C} 8-\mathrm{C} 9-\mathrm{C} 10 & 2.2(6) \\ \mathrm{C} 2-\mathrm{C} 3-\mathrm{C} 4-\mathrm{O} 3 & -83.3(4) & \mathrm{B} 2-\mathrm{C} 8-\mathrm{C} 9-\mathrm{C} 10 & -178.0(3) \\ \mathrm{Br} 1-\mathrm{C} 3-\mathrm{C} 4-\mathrm{O} 3 & 152.6(2) & \mathrm{O} 2-\mathrm{C} 6-\mathrm{C} 10-\mathrm{C} 11 & 179.7(3) \\ \mathrm{C} 2-\mathrm{C} 3-\mathrm{C} 4-\mathrm{C} 5 & 37.8(4) & \mathrm{C} 5-\mathrm{C} 6-\mathrm{C} 10-\mathrm{C} 11 & -1.0(6) \\ \mathrm{Br} 1-\mathrm{C} 3-\mathrm{C} 4-\mathrm{C} 5 & -86.2(3) & \mathrm{O} 2-\mathrm{C} 6-\mathrm{C} 10-\mathrm{C} 9 & -2.5(5) \\ \mathrm{O} 1-\mathrm{C} 1-\mathrm{C} 5-\mathrm{C} 6 & -177.2(3) & \mathrm{C} 5-\mathrm{C} 6-\mathrm{C} 10-\mathrm{C} 9 & 176.8(3) \\ \mathrm{C} 12-\mathrm{C} 1-\mathrm{C} 5-\mathrm{C} 6 & 0.5(6) & \mathrm{C} 8-\mathrm{C} 9-\mathrm{C} 10-\mathrm{C} 6 & -0.8(6) \\ \mathrm{O} 1-\mathrm{C} 1-\mathrm{C} 5-\mathrm{C} 4 & -1.0(6) & \mathrm{C} 8-\mathrm{C} 9-\mathrm{C} 10-\mathrm{C} 11 & 176.9(4) \\ \mathrm{C} 12-\mathrm{C} 1-\mathrm{C} 5-\mathrm{C} 4 & 176.8(3) & \mathrm{C} 6-\mathrm{C} 10-\mathrm{C} 11-\mathrm{C} 12 & 0.7(5) \\ \mathrm{O} 3-\mathrm{C} 4-\mathrm{C} 5-\mathrm{C} 1 & 111.1(4) & \mathrm{C} 9-\mathrm{C} 10-\mathrm{C} 11-\mathrm{C} 12 & -177.0(4) \\ \mathrm{C} 3-\mathrm{C} 4-\mathrm{C} 5-\mathrm{C} 1 & -10.6(5) & \mathrm{C} 10-\mathrm{C} 11-\mathrm{C} 12-\mathrm{C} 1 & 0.1(6) \\ \mathrm{O} 3-\mathrm{C} 4-\mathrm{C} 5-\mathrm{C} 6 & -72.7(4) & \mathrm{O} 1-\mathrm{C} 1-\mathrm{C} 12-\mathrm{C} 11 & 177.1(3) \\ \mathrm{C} 3-\mathrm{C} 4-\mathrm{C} 5-\mathrm{C} 6 & 165.6(3) & \mathrm{C} 5-\mathrm{C} 1-\mathrm{C} 12-\mathrm{C} 11 & -0.8(6) \\ \end{array}$

Hydrogen-bond geometry $\left(A,{ }^{\circ}\right)$

\begin{tabular}{lllll}
\hline$D-\mathrm{H} \cdots A$ & $D-\mathrm{H}$ & $\mathrm{H} \cdots A$ & $D \cdots A$ & $D-\mathrm{H} \cdots A$ \\
\hline $\mathrm{C} 11-\mathrm{H} 11 \cdots \mathrm{O} 4^{\mathrm{i}}$ & 0.93 & 2.57 & $3.188(6)$ & 124 \\
\hline
\end{tabular}

Symmetry code: (i) $x, y-1, z$. 